\title{
Nonequilibrium dislocation dynamics and instability of driven vortex lattices in two dimensions
}

\author{
Igor S. Aranson, ${ }^{1}$ Stefan Scheidl, ${ }^{1,2}$ and Valerii M. Vinokur ${ }^{1}$ \\ ${ }^{1}$ Materials Science Division, Argonne National Laboratory, Argonne, IL 60439 \\ ${ }^{2}$ Institut für Theoretische Physik, Universität zu Köln, Zülpicher Str. 7¥, D-50937 Köln, Germany
}

(January 29, 2018)

\begin{abstract}
We consider dislocations in a vortex lattice that is driven in a two-dimensional superconductor with random impurities. The structure and dynamics of dislocations is studied in this genuine nonequilibrium situation on the basis of a coarse-grained equation of motion for the displacement field. The presence of dislocations leads to a characteristic anisotropic distortion of the vortex density that is controlled by a Kardar-Parisi-Zhang nonlinearity in the coarse-grained equation of motion. This nonlinearity also implies a screening of the interaction between dislocations and thereby an instability of the vortex lattice to the proliferation of free dislocations.
\end{abstract}

PACS numbers: 74.60.Ge, 61.72.Bb

\section{INTRODUCTION}

Transport of periodic media such as vortex lattices in superconductors and charge-density waves through random environments plays a paradigmatic role in condensed matter physics. While the pinning dominated low-drive regime exhibiting glassy features has long been a subject of extensive research, the nontrivial properties of the high-velocity regime were recognized only recently. The prediction of a disorder induced nonequilibrium phase transition from plastic to coherent motion of the vortex lattice upon increasing drive, triggered extensive studies of rapidly driven disordered lattices and have attracted much recent interest in theory, 6 experiments, 09 and simulations 10 t 15

The observed dynamic transition can be described qualitatively as dynamic "melting" in analogy to the equilibrium melting transition, where disorder induces an effective "shaking" temperature 1 However, this analogy cannot be extended to the important questions regarding the role of disorder in structural transformations of periodic structures. The intrinsic nonequilibrium nature of the driven state renders the analysis of the structural transition specifically challenging. Even the equilibrium melting is described theoretically in the two-dimensional case only. Within the Kosterlitz-Thouless (KT) theory 16 melting is mediated by the unbinding of dislocation pairs. The underlying melting mechanisms are by far less understood in higher dimensions. Yet the issue of the stability of the ordered phase with respect to the formation of topological defects was identified as a key issue for the structural transitions, and substantial progress was achieved in understanding the role and contribution of disorder in the static case.17 Whereas in equilibrium the criteria for the stability of topological order follow from comparing relevant energy scales, the analysis of defect nucleation in the nonequilibrium situation is more subtle since it can no longer rely on energy balance considerations. In this paper we undertake a study of the stability of the topological order of the dynamic state focusing specifically on the two-dimensional vortex lattice driven through a disordered environment. We describe an intrinsic nonequilibrium mechanism giving rise to the proliferation of topological defects and therefore the instability of the driven vortex lattice.

The outline of this paper is as follows: in Sec. II we specify the coarse-grained equation of motion for the displacement field. In Sec. III we study the anisotropic and subdiffusive nature of the dynamics of a single dislocation neglecting the effect of the Kardar-Parisi-Zhang (KPZ) nonlinearity, which is discussed further in Sec. IV. There we show that the KPZ term leads to a "spiral" structure of dislocations, screening their long-range interaction, and recovering a normal linear diffusion of defects. Our results are summarized and discussed in Sec. V. Some technical aspects are deferred to the Appendix.

\section{MODEL}

We examine a dilute system of "test" dislocations embedded into the elastic medium of the two-dimensional vortex lattice. The vortices are driven along one of the principal lattice directions, the $x$ axis. The dynamics can be formulated in terms of a Langevin equation of motion for the displacement $u$ of vortices from their perfegt lattice positions that move with average velocity $v$. 18 The motion is governed by a competition of elastic interactions between vortices, thermal noise, and pining forces. Pinning is described by a potential $V$ with local correlation $\overline{V(\mathbf{R}) V(\mathbf{0})}=\Delta_{0} \delta(\mathbf{R})$, where the $\delta$ function is supposed to have a width of the order of the superconducting coherence length $\xi$.

Since the unbinding of dislocations is controlled by their interaction on large scales it is legitimate to use a coarse-grained description of the vortex lattice. It was derived recently 2 t 6 that on large spatial scales the equa- 
tion of motion assumes the form

$\eta \dot{u}=c \nabla^{2} u+\delta F+\zeta+\chi \nabla_{x} u+\frac{\lambda}{2}(\nabla u)^{2}+f(\mathbf{r}+\mathbf{v} t)$.

For our purposes it is sufficient to retain only the component of the displacement field parallel to the velocity. Although the other components also experience fluctuations on large scales, they can only further increase the instability of the lattice, which we find below to occur even in the absence of transverse displacements. Equation (11) is written in the frame moving with the average velocity of the vortices, where each vortex has a vanishing average displacement around its average position $\mathbf{r} . \eta$ is the vortex friction coefficient. For simplicity we consider the elastic interaction as uniform, i.e., we use only one elastic constant $c$, ignoring a distinction between shear and compression moduli and additional anisotropic corrections obtained from coarse graining. $\zeta$ is a thermal noise with temperature $T$. Although $\eta, c$ and $T$ are renormalized under coarse graining, the corrections are small in comparison to the original values for sufficiently large drift velocities. The stress coefficient $\chi$ and the Kardar-Parisi-Zhang (KPZ) nonlinearity $\lambda 19$ which are absent in the bare equation of motion, are generated under coarse graining. The pinning force $f$, which is simply a derivative of the pinning potential in the bare equation of motion, acquires a random-force character under renormalization. In principle, it depends on the displacement as $f(\mathbf{r}+\mathbf{v} t+\mathbf{u})$. However, this dependence can be neglected after the coarse-graining has been performed and $f$ has acquired random-force character. In the limit of large drift velocities these parameters take the following values: $t$

$$
\begin{aligned}
\chi & \approx \frac{\Delta_{0} c^{2}}{\xi^{3} a^{3} \eta^{3} v^{3}}, \\
\lambda & \approx \frac{\Delta_{0} c^{2}}{\xi^{4} a^{2} \eta^{3} v^{3}}, \\
\delta F & \approx \frac{\Delta_{0}}{\xi^{4} \eta v}, \\
\frac{\Delta_{0}^{2}}{f(\mathbf{R}) f(\mathbf{0})} & \approx \frac{\Delta^{2}}{\xi^{6} \eta^{2} v^{2}} \delta(\mathbf{R}), \\
\langle\zeta(\mathbf{r}, t) \zeta(\mathbf{0}, 0)\rangle & =2 \eta T \delta(\mathbf{r}) \delta(t) .
\end{aligned}
$$

$f$ and $\zeta$ are assumed to be Gaussian distributed with zero mean. $a$ is the vortex lattice spacing. The actual driving force $F=\eta v+\delta F$ required to achieve the prescribed velocity $v$ is determined from the consistency condition that the average displacement must vanish.

The linear stress term in Eq. (11) can actually be eliminated by a transformation of the displacement $\tilde{u}(\tilde{\mathbf{r}}, t)=$ $u(\tilde{\mathbf{r}}-(\chi / \eta) t \hat{\mathbf{x}}, t)$ to a new frame mqving with velocity $\tilde{v}=v-\chi / \eta$ in the laboratory frame. 20 The coordinates are related by $\tilde{\mathbf{r}}=\mathbf{r}+(t \chi / \eta) \hat{\mathbf{x}}$, where $\hat{\mathbf{x}}$ denotes the unit vector along the velocity direction. The following analysis is based on the transformed equation. For simplicity of notation we subsequently drop the tilde identifying transformed quantities.

\section{LINEAR PROBLEM $(\lambda=0)$}

We first examine the model in the absence of the KPZ nonlinearity to set the stage for its subsequent inclusion. In the above model dislocations are incorporated as a discontinuity in the displacement field of amplitude $a$, the vortex spacing. Due to the restriction of our consideration to the displacement component parallel to the velocity, the only possible orientations of Burgers vectors are parallel or antiparallel to the velocity.

In order to derive the dynamic response of a dislocation to the fluctuations of the elastic medium it is convenient to split the displacement field into a topologically nontrivial (multi-valued) part $u_{0}$ and a single-valued part $u_{\sim}$

$$
\begin{aligned}
u(\mathbf{r}, t) & =u_{0}\left(\mathbf{r}-\mathbf{r}_{\mathrm{d}}(t)\right)+u_{\sim}(\mathbf{r}, t), \\
u_{0}(\mathbf{r}) & \equiv \frac{a}{2 \pi} \varphi(\mathbf{r}) .
\end{aligned}
$$

The dislocation position is $\mathbf{r}_{\mathrm{d}}(t)$ and $\varphi(\mathbf{r})$ is the angle enclosed between the $x$ axis and $\mathbf{r}$. The problem at hand is then to derive an equation of motion for $u_{\sim}$ and $\mathbf{r}_{\mathrm{d}}$ from Eq. (11). To this end we consider for the moment $\mathbf{r}_{\mathrm{d}}(t)$ as given and find

$$
\eta \dot{u}_{\sim}-c \nabla^{2} u_{\sim}=\zeta+f(\mathbf{r}+\mathbf{v} t)-\eta \dot{\mathbf{r}}_{\mathrm{d}} \cdot \nabla u_{0}\left(\mathbf{r}-\mathbf{r}_{\mathrm{d}}\right) .
$$

Since this equation is linear in $u_{\sim}$ one can easily calculate $u_{\sim}$ for given $\zeta, f$, and dislocation trajectory $\mathbf{r}_{\mathrm{d}}$. Eventually we determine the dislocation velocity $\dot{\mathbf{r}}_{\mathrm{d}}$ in response to the forces $\zeta$ and $f$ from a condition of local equilibrium for the dislocation core,

$$
\nabla u_{\sim}\left(\mathbf{r}_{\mathrm{d}}(t), t\right)=0
$$

This equation is valid for "slow" changes of $u_{\sim}$ such that the displacement singularity "instantly" moves to a point where it is no longer subject to forces because its environment $u_{\sim}$ is locally homogeneous. $u_{\sim}$ can be directly obtained from Eq. (4) that has to be imposed with a short-scale cutoff of order $a$.

Supposing that the fluctuations of the elastic medium are weak and the dislocation displacement is slow, the equation of motion for the dislocation can be written in the form

$$
-i \omega \eta_{\mathrm{d}}(\omega) \mathbf{r}_{\mathrm{d}}(\omega)=\mathbf{f}_{\mathrm{d}}(\omega)
$$

with a Peach-Koehler-like force

$$
f_{\alpha}(t)=\epsilon_{\alpha \beta} \nabla_{\beta} u_{\sim}^{\mathrm{ext}}\left(\mathbf{r}_{\mathrm{d}}(t), t\right)
$$

( $\epsilon$ is the totally antisymmetric tensor; see the Appendix for some intermediate steps). Here $u_{\sim}^{\text {ext }}$ is the contribution to $u_{\sim}$ arising from the external forces $\zeta$ and $f$ at zero dislocation velocity. The dislocation drag coefficient 


$$
\begin{aligned}
\eta_{\mathrm{d}}(\omega) & =\frac{a}{2} \int_{\mathbf{k}} \frac{1}{-i \eta \omega+c k^{2}} \\
& \approx \frac{a \eta}{8 \pi c}\left(\ln \frac{4 \pi c}{\eta|\omega| a^{2}}+i \arctan \frac{4 \pi c}{\eta|\omega| a^{2}}\right) \text { for }|\omega| \ll \frac{4 \pi c}{\eta a^{2}}
\end{aligned}
$$

diverges logarithmically for small frequencies because of the long-ranged response of the displacement field to the dislocation motion. This divergence with vanishing frequency is equivalent to a divergence with increasing system size at zero frequency. It is characteristic for two dimensions and has been found previously for dislocations in yortex lattices retaining both displacement components21 and also in pattern forming systems.22.23

The force entering Eq. (6) has zero mean and correlations

$$
\begin{aligned}
\overline{\left\langle f_{\mathrm{d} \alpha}(\omega) f_{\mathrm{d} \beta}\left(\omega^{\prime}\right)\right\rangle} & =\delta\left(\omega+\omega^{\prime}\right) \Phi_{\alpha \beta}(\omega), \\
\Phi_{\alpha \beta}(\omega) & =\int_{\mathbf{k}} \tilde{k}_{\alpha} \tilde{k}_{\beta} \frac{2 \eta T+g \delta(\omega+\mathbf{k} \cdot \mathbf{v})}{\eta^{2} \omega^{2}+c^{2} k^{4}},
\end{aligned}
$$

where the disorder contribution is proportional to $g \equiv$ $\Delta_{0}^{2} / \xi^{6} \eta^{2} v^{2}$ and $\tilde{k}_{\alpha} \equiv \sum_{\beta} \epsilon_{\alpha \beta} k_{\beta}$. It is instructive to compare the relative strength of thermal and pinning contributions

$$
\begin{aligned}
& \Phi_{\alpha \beta}^{\mathrm{th}}(\omega) \approx \delta_{\alpha \beta} \frac{\eta T}{8 \pi c^{2}} \ln \left[1+\left(\frac{4 \pi c}{\eta \omega a^{2}}\right)^{2}\right], \\
& \Phi_{y y}^{\mathrm{pin}}(\omega) \approx \frac{g}{6 \pi^{2} \eta^{2} v^{3}}\left|\frac{\eta \omega}{c}\right|^{1 / 2} \text { for }|\omega| \ll \frac{\pi^{2} c}{\eta a^{2}}, \\
& \Phi_{x x}^{\mathrm{pin}}(\omega) \approx \frac{g}{4 \pi^{2} c^{2} v}\left|\frac{c}{\eta \omega}\right|^{1 / 2} \text { for }|\omega| \ll \frac{\pi^{2} c}{\eta a^{2}} .
\end{aligned}
$$

At low frequency the pinning contribution to the $y$ component is negligible in comparison to the thermal contribution, $\Phi_{y y}^{\mathrm{pin}}(\omega) \ll \Phi_{y y}^{\mathrm{th}}(\omega)$. However, the pinning contribution to the $x$-component dominates over the thermal contribution, $\Phi_{x x}^{\mathrm{pin}}(\omega) \gg \Phi_{x x}^{\mathrm{th}}(\omega)$.

Since $\mathbf{f}_{\mathrm{d}}$ vanishes on average, the dislocation has mean zero velocity in the frame of Eq. (6), i.e., their velocity is smaller than that of the vortices by $\chi / \eta \propto v^{-3}$. The diffusive behavior of dislocations is readily found from Eq. (6). In the absence of disorder dislocations actually move subdiffusively,

$$
\left\langle\left[r_{\mathrm{d} \alpha}(t)-r_{\mathrm{d} \alpha}(0)\right]^{2}\right\rangle \approx \frac{32 T}{a^{2} \eta} \frac{|t|}{\ln \left(4 \pi c|t| / \eta a^{2}\right)}
$$

for $|t| \gg \eta a^{2} / 4 \pi c$. Despite of this peculiar dynamic behavior the fluctuation-dissipation theorem holds for dislocations in the absence of disorder, which implies that dislocation pairs nevertheless unbind at the KT transition temperature.

The presence of disorder can significantly affect the diffusive behavior of the dislocation. For qualitative purposes we may still use the linear response approach, where the forces acting on the dislocation are evaluated at the undisplaced dislocation position. This approach (shguld provide a good approximation for a considerable time interval because of the logarithmic divergence of the drag coefficient. From the above equations one finds that (\$ke motion is superdiffusive along the $x$-direction,

$$
\overline{\left\langle\left[x_{\mathrm{d}}(t)-x_{\mathrm{d}}(0)\right]^{2}\right\rangle} \approx \frac{64 g c^{1 / 2}}{3 \pi v \eta^{5 / 2} a^{2}} \frac{|t|^{3 / 2}}{\ln ^{2}\left(4 \pi c|t| / \eta a^{2}\right)}
$$

on large time scales $|t| \gg \eta^{3} v^{2} T^{2} / c g^{2}$, where the pinning contribution dominates over the thermal contribution. The validity of Eq. (12) is limited by the fact that the nonlinear terms in the equation of motion for $\mathbf{r}_{\mathrm{d}}$ have been neglected. This could lead on largest time scales to a further renormalization of the dislocation velocity $\tilde{v}$. However, a quantitative calculation of the dislocation velocity is beyond the scope of the present paper, since it would require the inclusion of additional contributions to the equation of motion, such as those due to Peierls barriers. However, despite of the possibility that Eq. (12) may not capture the true large-scale behavior one may conclude from a comparison of Eq. (12) to Eq. (11) that the shaking effects of disorder correspond on large scales to an effectively infinite temperature. This result provides a first indication for a disorder-driven unbinding of dislocations.

\section{NONLINEAR PROBLEM $(\lambda=0)$}

So far the quadratic KPZ nonlinearity has been excluded from our analysis and now we address the question how it modifies the above findings. For simplicity, we initially drop the random force term and the thermal noise to examine the structure and dynamics of single dislocations as well as the interaction between dislocations. In this case Eq. (11) is reduced to the Burgers equation

$$
\eta \dot{u}=c \nabla^{2} u+\frac{\lambda}{2}(\nabla u)^{2}+\delta F .
$$

In analogy to the usual pinning problem we consider the vortex drift velocity, which enters the last equation through $\lambda$, as prescribed and determine the related force contribution $\delta F$ from the stationarity condition $\dot{u}=0$. Topological defects in Eq. (13) were stulied recently in the context of pattern-forming systems, 2328 where they constitute the source of "spiral waves." Therefore it is possible to carry over part of the previously achieved analysis to the present context.

It is convenient to perform the well-known Hopf-Cole transformation that leads to a linear equation for the function $W \equiv \exp (\lambda u / 2 c)$,

$$
\eta \dot{W}=c \nabla^{2} W-c k_{0}^{2} W
$$

Looking for a stationary solution, the constant

$$
k_{0}^{2}=-\frac{\lambda}{2 c^{2}} \delta F
$$


will be determined from the condition that the solution is not singular at the core (for the simplest choice $k_{0}=0$ the solution $W$ exhibits a non-physical $r^{-1}$ singularity at $r=0)$. Note, that the stationarity of $u$ implies that $\lambda$ and $\delta F$ must have opposite sign.

Equation (13) possesses a solution of the form

$$
u(\mathbf{r})=\frac{a}{2 \pi} \varphi(\mathbf{r})+\mu(r)
$$

where $\mu(r)$ is a rotation-symmetric contribution induced by the KPZ nonlinearity. This solution still represents a topological defect with the characteristic multivaluedness of the displacement $u(\varphi+2 \pi m)=u(\varphi)+a m$ (our explicit considerations apply to $m=1$ only, the generalization to integer $m$ is straightforward). The angular and radial dependences of $W$ factorize,

$$
W_{0}(r, \varphi)=\exp [(a \lambda / 4 \pi c) \varphi] w(r)
$$

and for a stationary solution of Eq. (14) the radial contribution $w(r)=\exp [\lambda \mu(r) / 2 c]$ has to satisfy a modified Bessel equation

$$
\partial_{r}^{2} w+\frac{1}{r} \partial_{r} w+\frac{\alpha^{2}}{r^{2}} w=k_{0}^{2} w
$$

where $\alpha \equiv a \lambda / 4 \pi c$. Thus the solution to Eq. (18) is a modified Bessel function $w(r)=K_{i \alpha}\left(k_{0} r\right)$ with imaginary index.29 This solution has two characteristic length scales separating three regions. On large length scales $r \gg L_{s} \equiv k_{0}^{-1}$ this solution decays exponentially, $w(r) \propto$ $\exp \left[-k_{0} r\right] / r^{1 / 2}$. For $r \ll k_{0}^{-1}$ and $\alpha \ll 1$ an expansion of the Bessel function yields $w(r) \approx \sin \left[\alpha\left(\ln \left(2 / k_{0} r\right)-\gamma\right)\right]$ with Euler's constant $\gamma$. Thus $w(r)$ assumes a maximum at a second characteristic scale

$$
r_{0} \equiv k_{0}^{-1} e^{-\pi / 2 \alpha}
$$

For $r \ll r_{0}$ the solution becomes strongly oscillating, which is unphysical. It is important to keep in mind that the equation of motion for the displacement field is valid only on scales larger than a cutoff scale $R_{c}$ of the order of the vortex spacing. Therefore the oscillatory behavior of $w$ at small scales is an artifact of Eq. (13) that does not account for the dislocation core structure. The above solution is physically meaningful only as long as $\mu(r)$ or $w(r)$ depend monotonously on the distance, i.e., the outermost maximum of the solution found above has to be identified with the core radius. Thus Eq. (19) actually determines $k_{0}$, or equivalently the screening length $L_{s}$ as a function of $\alpha, 30$

$$
\begin{aligned}
L_{s} & =k_{0}^{-1} \sim R_{c} e^{\pi / 2 \alpha} \sim a e^{\left(v / v_{0}\right)^{3}}, \\
v_{0}^{3} & =\frac{\Delta_{0} c}{2 \pi^{2} \xi^{4} a^{3} \eta^{3}} .
\end{aligned}
$$

For small $\alpha$, i.e., large velocity $v$ of the vortex lattice, $L_{s}$ is exponentially large. Since for $r \rightarrow \infty$ the solution $w \sim \exp \left[-k_{0} r\right] / r^{1 / 2}$ has exponential asymptotics, the displacement $u \approx(2 c / \lambda) \ln w \approx-(2 c / \lambda) k_{0} r$ increases proportional to the distance from the dislocation core.

The exponential decay of the function $K$ readily implies exponential screening of the interaction between several dislocations at positions $\mathbf{r}_{i}$ with distances large compared to $L_{s}$. For pattern forming systems this screening was a subject of intensive investigations 2527 Indeed, since Eq. (14) is linear in $W$, the multi-dislocation solution can be approximated as a linear superposition of individual solutions,

$$
W(\mathbf{r})=\sum_{i} W_{0}\left(\left|\mathbf{r}-\mathbf{r}_{\mathbf{i}}\right|\right)+\tilde{w}
$$

where the correction $\tilde{w}$ is introduced in order to fix "topological conditions" imposed by the field $u$. For a wellseparated ensemble of dislocations the overlap between the individual contributions to $W$ is exponentially small. From the velocity of a dislocation under the influence of other dislocations one can actually obtain the dislocation interaction. The resulting interaction between two dislocations $i$ and $j$ decays as $\exp \left[-2 k_{0}\left|\mathbf{r}_{\mathbf{i}}-\mathbf{r}_{\mathbf{j}}\right|\right]$ for $k_{0}\left|\mathbf{r}_{\mathbf{i}}-\mathbf{r}_{\mathbf{j}}\right| \gg 1$ (for $k_{0}\left|\mathbf{r}_{\mathbf{i}}-\mathbf{r}_{\mathbf{j}}\right|<$ there is a crossover to the usual power-like behavior).26.31

The structure of such a "spiral" dislocation is illustrated schematically in Fig. 11. Note that the lattice is compressed in front of the dislocation, whereas it is diluted behind the dislocation. This effect is independent of the orientation of the Burgers vector parallel or antiparallel with the velocity. Given the fact that $\lambda$ is positive this effect can be easily understood from Eq. (13), if one considers the KPZ nonlinearity as perturbation to the usual dislocation: the displacement strains are largest close to the dislocation core. Therefore the vortices close to the core experience a force that drives them further in the direction of the velocity until the elastic forces establish force equilibrium in a configuration, where all vortices move with the same velocity $v$.

Due to the presence of the KPZ term in the equation of motion (13) the force required to achieve a vortex motion with velocity $v$ is reduced: $\delta F=F-\eta v=-(2 c / \lambda) k_{0}^{2}<$ 0 . However, this contribution of the KPZ term, which is generated by disorder, actually represents only a further correction to the immediate pinning force (2c), which is positive. For large $v$ the correction due to the KPZ term is negligible in comparison to the pinning force, since the former contribution decays exponentially for large $v$ whereas the latter decays only algebraically. Considering both contributions consistently together, one finds $F>\eta v$, i.e., even in the presence of the KPZ nonlinearity disorder actually slows down the vortex motion.

The structure of the vortex lattice in the presence of a dislocation/antidislocation pair is illustrated in Fig. 2. This structure was calculated numerically directly from Eq. (13) using a lattice domain with periodic boundary conditions. In Fig. 2 two such domains are reproduced. The inhomogeneity of the density is apparent and the 
density can change along narrow boundaries that correspond to shock wave fronts.19

Let us now include the effect of weak thermal noise and disorder in the dynamics of a single dislocation. After the Hopf-Cole transformation Eq. (11) assumes the form

$$
\eta \dot{W}=c \nabla^{2} W-c k_{0}^{2} W+\frac{\lambda}{2 c}[\zeta+f(\mathbf{r}+\mathbf{v} t)] W
$$

To derive the resulting equation of motion for a dislocation we apply a perturbation technique, 26 where the force fields of thermal noise and disorder are projected on the translation modes $W_{x, y}$ of the unperturbed Eq. (22) (see also the Appendix). Since the translation mode is simply $W_{x, y}=\partial_{x, y} W_{0}$, the equation of motion for the dislocation position $\mathbf{r}_{\mathbf{0}}$ is of the form

$$
\begin{aligned}
\eta_{\mathrm{d}} \partial_{t} \mathbf{r}_{\mathbf{0}}= & \frac{\lambda}{2 c} \int d^{2} r^{\prime}\left[\zeta\left(\mathbf{r}^{\prime}, t\right)+f\left(\mathbf{r}^{\prime}-\mathbf{r}_{0}(t)+\mathbf{v} t\right)\right] \\
& \times W_{0}\left(\mathbf{r}^{\prime}\right) \nabla W_{0}\left(\mathbf{r}^{\prime}\right)
\end{aligned}
$$

with an effective drag coefficient $\eta_{\mathrm{d}}$ of the form

$$
\eta_{\mathrm{d}}=\frac{\eta}{2} \int d^{2} r^{\prime}\left[\nabla W_{0}\left(\mathbf{r}^{\prime}\right)\right]^{2}
$$

Because of the exponential localization of the function $W_{0}$, the drag coefficient $\eta_{\mathrm{d}}$ is finite, in contrast to the linear case, where it diverges logarithmically with the volume. From Eq. (23) we obtain the mean squared displacement [abbreviating $\zeta^{(n)} \equiv \zeta\left(\mathbf{r}^{(n)}, t^{(n)}\right)$ and $f^{(n)} \equiv$ $\left.f\left(\mathbf{r}^{(n)}-\mathbf{r}_{0}\left(t^{(n)}\right)+\mathbf{v} t^{(n)}\right)\right]$

$$
\begin{aligned}
\left\langle\mathbf{r}_{0}^{2}(t)\right\rangle= & \frac{\lambda^{2}}{4 c^{2} \eta_{d}} \int_{0}^{t} d t^{\prime} \int_{0}^{t} d t^{\prime \prime} \int d^{2} r^{\prime} d^{2} r^{\prime \prime}\left\langle\left[\zeta^{\prime}+f^{\prime}\right]\left[\zeta^{\prime \prime}+f^{\prime \prime}\right]\right\rangle \\
& \times W_{0}\left(\mathbf{r}^{\prime}\right) \nabla W_{0}\left(\mathbf{r}^{\prime \prime}\right) W_{0}\left(\mathbf{r}^{\prime \prime}\right) \nabla W_{0}\left(\mathbf{r}^{\prime \prime}\right)
\end{aligned}
$$

Let us first consider only the effect of thermal noise $\zeta$. Averaging Eq. (25) and utilizing the exponential decay of $W$ at large $r$, we readily obtain a normal diffusive behavior,

$$
\left\langle\mathbf{r}_{0}^{2}(t)\right\rangle \sim t
$$

Thus, the exponential screening of the dislocation field due to the KPZ nonlinearity is responsible for the transition from subdiffusion [Eq. (11)] to normal diffusion.

To include the effect of quenched disorder we have to perform an additional disorder averaging of Eq. (25). Thereby we have to assume that the position of the dislocation is not correlated with the disorder, which is true at large enough drift velocity where the dislocation moves with the vortices. As in the case of purely thermal noise we obtain normal diffusion of the dislocation due to the exponential localization of $W$, in contrast to superdiffusion in the linear case.

\section{CONCLUSIONS}

The most important implication of the exponential screening of the dislocation interaction is that even arbitrarily weak thermal noise or random force result in an unbinding of dislocations (see Ref. 28). This means that the corresponding Kosterlitz-Thouless temperature is zero in this situation, and that the topological order of the vortex lattice is always destroyed on largest scales. While previous arguments in favor of such an instability were based on scaling arguments 2 and numerical simulation 14 we have presented here an intrinsic nonequilibrium mechanism. This instability mechanism is primarily based on the presence of the KPZ nonlinearity in the equation of motion, which is generated by coarse graining the equation of motion of a driven vortex lattice.

At this point we would like to recall that our analysis was based on the simplified model with only one displacement component. Fluctuations of the second component will probably lead to a significant further reduction of the stability of the vortex lattice, i.e., the true screening length could possibly be smaller than the value for $L_{s}$ calculated above.

It is instructive to compare the dislocation screening length $L_{s}$ to the crossover scale where the KPZ nonlinearity becomes relevant for thermal fluctuations of the displacement field in the absence of dislocations. The latter scale is $\xi_{c} \equiv \exp \left(8 \pi c^{3} / \eta T \lambda^{2}\right), 32$ i.e., it is also exponentially large for small $\lambda$. However, the functional dependence of $\lambda$ is different and $\mathrm{E}_{s} \ll \xi_{c}$ for small $\lambda$. [Note, that $\xi_{c}$ is defined only for finite temperature while $L_{s}$ is well-defined even for zero temperature.] Therefore, dislocations become important for the structure of the system before it can show a crossover to the strong-coupling behavior of the elastic system.

In a remote analogy the interaction of dislocation pairs in a driven vortex lattice can be compared to the interaction of vortex pairs in a superconducting film. In the former case screening is induced by disorder, which in the latter case is provided by magnetic fields. In the absence of screening both systems would perform a KosterlitzThouless transition. Screening suppresses this transition as a large-scale phenomenon. However, for large enough screening lengths this crossover can still be well pronounced. The screening length of the vortex interaction in films is known to be macroscopic. The screening length of dislocations $L_{s}$ as found above will also be macroscopic for $v \gg v_{0}$ because of its exponential increase with the vortex drift velocity. It is therefore possible that the lattice may be well ordered on experimentally relevant scales and that a dynamic melting or freezing can still be found as a pronounced crossover.

In the nonequilibrium mechanism for the dislocation unbinding examined above the KPZ nonlinearity played a major role. It is worthwhile to point out that even if dislocations are artificially suppressed in a purely elastic 
approach, this nonlinearity can induce dynamic transitions between "rough" and "flat" sliding phases. 33 The above analysis demonstrates that even the "flat" sliding phase is actually penetrated by free dislocations on length scales beyond $L_{s}$. Therefore one might suspect that their presence modifies the nature of this roughnening transition or even blurs the transition, turning it into a mere crossover. Again, this crossover may yet be observable because $L_{s}$ grows exponentially for large drift velocities.

To conclude, we have found an instability of the simplified one-component model of a drifting vortex lattice to proliferation of free defects. As already mentioned above, the presence of a second displacement component can only lead to a further increase of fluctuations and to a reduction of the instability. We have presented an intrinsic nonequilibrium mechanism for such an instability based on the presence of the KPZ nonlinearity in the coarse-grained equation of motion for the displacement field of a vortex lattice driven in a disordered environment. The instability is due to an exponential screening of the dislocation interaction on a scale $L_{s}$ that increases exponentially with the drift velocity of the vortex lattice.

This work was supported by Argonne National Laboratory through the U.S. Department of Energy, BESMaterials Sciences, under contract No. W-31-109-ENG38 and by the NSF Science and Technology Center for Superconductivity under contract No. DMR91-20000. S. S. acknowledges support from the Deutsche Forschungsgemeinschaft under Project No. SFB341 and Grant No. SCHE/513/2-1.

\section{APPENDIX:}

In this appendix we give some intermediate steps of the calculation leading to equation of motion (6) for the dislocation in the linear medium. Furthermore we relate this calculation to a projection of the force field onto the translation modes of the dislocation, as used in Sec. IV.

For given $\mathbf{r}_{\mathrm{d}}$ Eq. (1) can be solved for $u_{\sim}$. Equation (5) then implies

$$
\begin{aligned}
0 & =\epsilon_{\alpha \beta} \nabla_{\beta} u_{\sim}\left(\mathbf{r}_{\mathrm{d}}(t), t\right) \\
& =\epsilon_{\alpha \beta} \int d^{2} r^{\prime} d t^{\prime} \nabla_{\beta} G\left(\mathbf{r}_{\mathrm{d}}(t)-\mathbf{r}^{\prime}, t-t^{\prime}\right) \\
& \times\left[\zeta\left(\mathbf{r}^{\prime}, t^{\prime}\right)+f\left(\mathbf{r}^{\prime}+\mathbf{v} t^{\prime}\right)-\eta \dot{\mathbf{r}}_{\mathrm{d}}\left(t^{\prime}\right) \cdot \nabla u_{0}\left(\mathbf{r}^{\prime}-\mathbf{r}_{\mathrm{d}}\left(t^{\prime}\right)\right)\right]
\end{aligned}
$$

where $G$ is the Greens function for $u_{\sim}$. In linear response, $\zeta, f$, and hence $\dot{\mathbf{r}}_{\mathrm{d}}$ are small and one may replace $\mathbf{r}_{\mathrm{d}}\left(t^{\prime}\right)$ by $\mathbf{r}_{\mathrm{d}}(t)$ in the previous Equation. This equation has to be understood as force balance between the contribution $\mathbf{f}_{\mathrm{d}}$ arising from the external force fields $\zeta$ and $f$ and the friction force $\eta_{d} \dot{\mathbf{r}}_{\mathrm{d}}$. The separation of these contribution leads to Eq. (16).

Let us now briefly discuss the connection between the approaches used in Secs. III] and IV to calculate the dislocation dynamics. The subdiffusive behavior of dislocations in the linear system can be easily obtained from the equation of motion, taking into account that in the linear system (4) the translation mode is $u_{x y}=\partial_{x, y} u_{0} \sim 1 / r$. Thus, the effective mass diverges as $\ln r$. For the meansquared displacement we obtain (dropping the disorder term)

$$
\begin{aligned}
\left\langle\mathbf{r}_{0}^{2}(t)\right\rangle= & \frac{1}{\eta_{d}} \int_{0}^{t} \int_{0}^{t} \int d^{2} r^{\prime} d^{2} r^{\prime \prime} d t^{\prime} d t^{\prime \prime} \\
& \times\left\langle\zeta^{\prime} \zeta^{\prime \prime} \nabla u_{0}\left(\mathbf{r}^{\prime}\right) \nabla u_{0}\left(\mathbf{r}^{\prime \prime}\right)\right\rangle
\end{aligned}
$$

instead Eq. (25). After averaging we obtain $\left\langle\mathbf{r}_{0}^{2}(t)\right\rangle \sim$ $t / \ln r \approx t / \ln t$, which coincides with Eq. (11).

${ }^{1}$ A. E. Koshelev and V. M. Vinokur, Phys. Rev. Lett. 73, 3580 (1994).

${ }^{2}$ L. Balents and M. P. A. Fisher, Phys. Rev. Lett. 75, 4270 (1995).

${ }^{3}$ T. Giamarchi and P. Le Doussal, Phys. Rev. Lett. 76, 3408 (1996); L. Balents and M. C. Marchetti and L. Radzihovsky, Phys. Rev. Lett. 78, 751 (1997); T. Giamarchi and P. Le Doussal, Phys. Rev. Lett. 78, 752 (1997).

${ }^{4}$ S. Scheidl and V. M. Vinokur, Phys. Rev. E 57, 2574 (1998).

${ }^{5}$ L. Balents, M. C. Marchetti, and L. Radzihovsky, Phys. Rev. B 57, 7705 (1998).

${ }^{6}$ P. Le Doussal and T. Giamarchi, Phys. Rev. B 57, 11356 (1998).

7 S. Bhattacharya and M. J. Higgins, Phys. Rev. Lett. 70, 2617 (1993).

${ }^{8}$ M. C. Hellerqvist, D. Ephron, W. R. White, M. R. Beasley, and A. Kapitulnik, Phys. Rev. Lett. 76, 4022 (1996).

${ }^{9}$ C. D. Keener, M. L. Travick, S. M. Amirata, S. E. Hebboul, and J. C. Garland, Phys. Rev. Lett. 78, 1118 (1997).

${ }^{10}$ K. Moon, R. T. Scalettar and G. T. Zimányi, Phys. Rev. Lett. 77, 2778 (1996).

${ }^{11}$ M. C. Faleski, M. C. Marchetti, and A. A. Middleton, Phys. Rev. B 54, 12427 (1996).

12 S. Ryu, M. Hellerqvist, S. Doniach, A. Kapitulnik and D. Stroud, Phys. Rev. Lett. 77, 5114 (1996).

13 D. Domínguez, N. Grønbech-Jensen, and A. R. Bishop, Phys. Rev. Lett. 78, 2644 (1997).

${ }^{14}$ S. Spencer and H. J. Jensen, Phys. Rev. B 55, 8473 (1997).

15 A. van Otterlo, R. T. Scalettar, G. T. Zimányi, R. Olsson, A. Petrean, W. Kwok, and V. Vinokur (unpublished).

${ }^{16}$ J. M. Kosterlitz and D. J. Thouless, J. Phys. C 6, 1181 (1973).

17 J. Kierfeld, T. Nattermann and T. Hwa, Phys. Rev. B 55, 626 (1997).

${ }^{18}$ We follow the notations of Ref. 4

${ }^{19}$ M. Kardar, G. Parisi, and Y.-C. Zhang, Phys. Rev. Lett. 56, 889 (1986).

${ }^{20}$ Note that this transformation can be performed only neglecting the discreteness of the lattice. The discreteness can be important at temperatures small compared to the 
Peierls barriers for dislocation motion; see also S. Scheidl and V.M. Vinokur, Phys. Rev. B 56, R8522 (1997).

${ }^{21}$ E. H. Brandt, Phys. Stat. Sol. 36, K167 (1969).

${ }^{22}$ E. D. Siggia and A. Zippelius, Phys. Rev. A 24, 1036 (1981).

${ }^{23}$ E. Bodenschatz, W. Pesch, and L. Kramer, Physica D 32 , 135 (1988).

${ }^{24}$ P. Hagan, SIAM J. Appl. Math. 42, 762 (1982).

${ }^{25}$ I. Aranson, L. Kramer and A. Weber, Physica D 53, 376384 (1991).

${ }^{26}$ I. Aranson, L. Kramer and A. Weber, Phys. Rev. E 47 3231 (1993).

${ }^{27}$ A. Nepomnyaschii and L. Pismen, Physica D 54, 183 (1992).

${ }^{28}$ I. S. Aranson, H. Chaté, and L.-H. Tang, Phys. Rev. Lett. 80, 2646 (1998).

29 The Bessel function $I$, which is also a solution to Eq. (18), cannot satisfy this condition at the core of the dislocation, see Ref. 24 for details.

${ }^{30}$ Equation (13) can be regularized by a Ginzburg-Landau equation with complex coefficients. Within this scheme, where one can calculate the actual dislocation core structure and verify the matching pocedure at the core radius and the resulting relation 20.24

31 The analysis of the complex Ginzburg-Landau equation in Ref. 26 suggests that the interaction between dislocations separated by a distance $r \gg k_{0}^{-1}$ is always repulsive regardless of the charges, since the core of the dislocation is exponentially screened. Oppositely-charged dislocation pairs recover attractive interaction at smaller distances. We expect the same interaction law also for dislocations in vortex lattice, since the particular structure of the core is not relevant for the large-scale dynamics.

${ }^{32}$ L.-H. Tang, T. Nattermann, and B. M. Forrest, Phys. Rev. Lett. 65, 2422 (1990).

${ }^{33}$ L.-W. Chen, L. Balents, M. P. A. Fisher, and M. C. Marchetti, Phys. Rev. B 96, 12798 (1996).

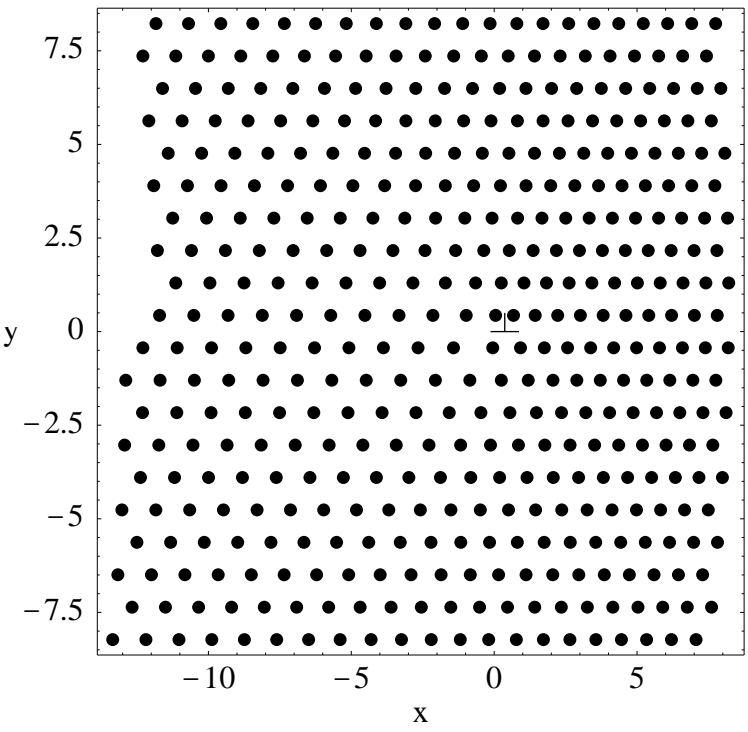

FIG. 1. Schematic illustration of the structure of a single dislocation (marked by the symbol $\perp$ ) in the vortex lattice driven along the $x$ axis (lengths are in units of $a$ ). The density is increased/reduced in directions in front of/behind the dislocation.

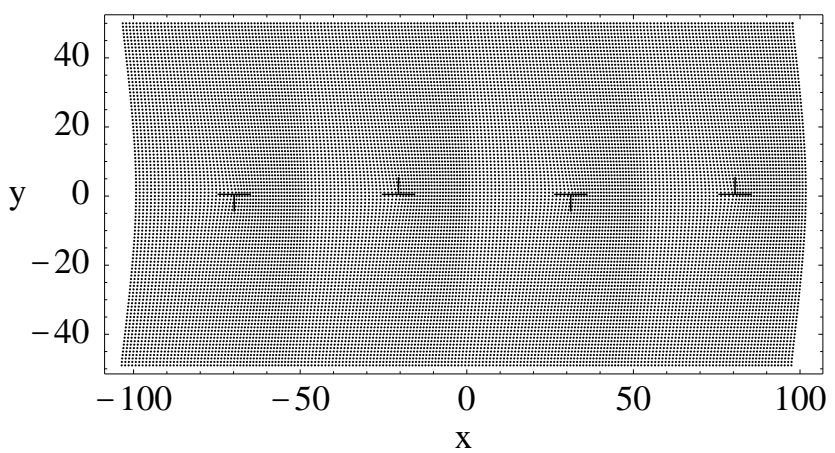

FIG. 2. Schematic illustration of a dislocation pair (repeating two unit cells with periodic boundary conditions) in the driven vortex lattice. The vortex density changes quite abruptly along pronounced shock wave fronts. The vortex lattice is shown here in a square lattice to make the local rotations and compression of the unit cell graphically more transparent although in reality the vortex lattice is hexagonal. 